Variations sur la lettre, le mètre et la mesure :

Shakespeare

\title{
La Reine des fées et l'idée du livre
}

\section{Bernard Tannier}

\section{(2) OpenEdition}

Journals

Édition électronique

URL : http://journals.openedition.org/shakespeare/978

DOI : $10.4000 /$ shakespeare. 978

ISSN : 2271-6424

Éditeur

Société Française Shakespeare

Édition imprimée

Date de publication : 1 novembre 1996

Pagination : 127-138

Référence électronique

Bernard Tannier, "La Reine des fées et l'idée du livre », Actes des congrès de la Société française

Shakespeare [En ligne], 14 | 1996, mis en ligne le 01 janvier 2007, consulté le 27 avril 2019. URL : http:// journals.openedition.org/shakespeare/978; DOI : 10.4000/shakespeare.978 


\section{Variations sur la lettre,}

le mètre et la mesure

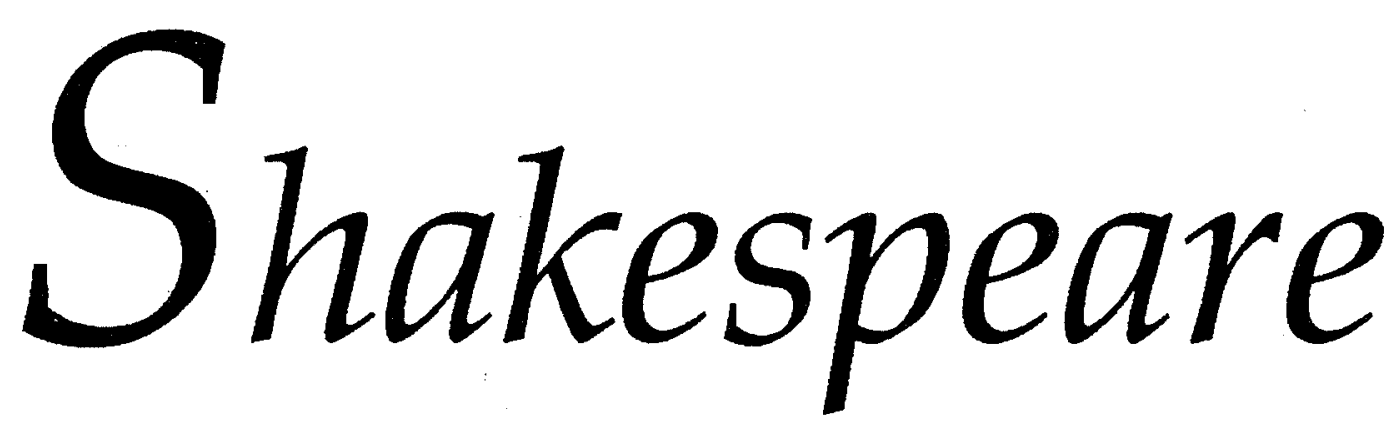

textes présentés par Dominique Goy-Blanquet

Directeur de la publication : Richard Marienstras 


\section{La Reine des fées et l'idée du livre}

Né en 1552, une centaine d'années environ après l'apparition du livre imprimé, Spenser donne plusieurs illustrations à "l'idée du livre" : d'abord son art d'utiliser les ressources de la nouvelle invention pour la présentation, voire l'élucidation partielle, de ses textes, et la politique auto-promotionnelle qui a présidé à leur publication; ensuite l'usage dans sa narration d'écrits fictifs sous forme de rouleaux de parchemin, de livres manuscrits, peut-être même de livres imprimés, et d'allusions à la production de ces textes; enfin l'idée qui a présidé à l'élaboration du poème, ce que Sidney appelle "fore-conceit" dans A Defence of Poesie ${ }^{1}$, que Spenser lui-même nomme "dark conceit" et qu'il prétend nous aider à déchiffrer grâce à un appareil d'appendices, idée soulignée par le symbolisme numérologique du nombre de livres annoncés et celui de livres réellement écrits.

De la première æuvre importante de Spenser, The Shepheardes Calender, parue en 1579 sous le nom d'auteur d'Immerito, Andrew Hadfield écrit, citant un article de McCanles : "Dans sa forme concrète, ce poème, accompagné de toute sa panoplie d'explications, de gloses et de commentaires, apparaît comme l'imitation fictive d'une édition de textes classiques par des humanistes"2. McCanles ajoute, citant Walter Ong, que ce type de livre, dans sa présentation concrète, avec "ses alignements et ses séparations spatiales", n'a pu voir le jour qu'avec l'imprimerie ${ }^{3}$.

L'appareil critique (attribué à un certain E. K.) présente le Shepheardes Calender à la fois comme un livre d'églogues du XVIe siècle "qui cache son sens derrière une façade de conventions pastorales et un language archaïque" mais aussi comme un monument à "ces conventions et à ce language"4. Or Spenser, pour mieux souligner son intention d'archaïsme, tente aussi de faire revivre certaines formes du livre déjà considérées comme dépassées. Par exemple, comme le souligne R. S. Luborsky, Spenser exploite l'archaïsme des gravures sur bois, dans le style de celles du Calendrier et Compost des Bergiers, alors que ce type d'illustration avait été "supplanté par des gravures sur cuivre". Il "imite aussi les manuscrits et les premiers incunables" en utilisant des "initiales décoratives". Enfin, il s'inspire des éléments de présentation de livres d'emblèmes, de livres de fables illustrés, ainsi que d'éditions de classiques, auxquels il se réfère dans ses gloses comme appartenant déjà au Passé, bien que ne datant que du début du XVI $\mathrm{Xiècle}^{5}$. Spenser exploite donc toutes les possibilités typographiques, y compris le recours à des formes déjà démodées, pour rendre sensible ce qui est le thème majeur de la pastorale, à savoir le passage du temps et la nostalgie de l'Age d'Or.

Non content d'utiliser toutes les possibilités de composition typographiques offertes par l'invention de Gutenberg, il s'est livré à une véritable stratégie en 
matière de publication pour créer son personnage de "New Poet", ainsi que le nomme E. K. Déjà dans le Shepheardes Calender, il avait utilisé la préface et diverses notes pour se poser comme l'auteur d'un certain nombre d'œuvres, apparemment fictives, soit attendant l'impression soit en gestation: "his Dreames, his Legendes, his Court of Cupide, and sondry others" ainsi qu'un traité de poétique : "The English Poet"6. Or, presque simultanément, en 1580, il faisait paraître deux fascicules contenant des lettres échangées avec Gabriel Harvey, qui ont pu être qualifiées par Hadfield de "self-promotional manifestos"7. D'autres œuvres lui étaient également attribuées : un "Epithalamion Thamesis", un "Dying Pellicane", des "Stemmata Dudleyana" et "Nine Comœdies", outre une mention de la Faerie Queene. D'autre part était souligné le fait que Spenser appartenait à l'entourage immédiat du duc de Leicester, qu'il avait été introduit à la cour, et qu'il entretenait des rapports de familiarité avec Philip Sidney. Harvey parle même d'un "areopagus" constitué de Sidney, Edward Dyer et Spenser, qui se consacrait à des essais de poésie mesurée. William Wilson souligne qu' "aucun Poète anglais n'avait jamais été annoncé d'une façon aussi ostentatoire"".

Tous ces efforts, s'ils assurèrent à Spenser sa réputation de "new poet" auprès d'un cercle restreint, ne lui valurent pas, pour des raisons politiques ${ }^{10}$, la promotion qu'il espérait. Il n'obtint qu'un poste de secrétaire auprès de Lord Grey of Wilton, gouverneur d'Irlande, lieu où il résidera désormais. Il reviendra néanmoins trois fois en Angleterre et y fera imprimer la Faerie Queene : les trois premiers livres en 1590, puis une édition regroupant ces trois premiers livres et les trois suivants en 1596. Une édition posthume, en 1609, reprendra celle de 1596 avec des fragments d'un septième livre. Parallèlement, il publiera des poèmes satiriques témoignant de sa déception de la cour et, indirectement, de la reine : le recueil de Complaints en 1591, et Colin Clout Comes Home Again en 1595.

Walter Ong, se référant à la première édition de la Faerie Queene, parle de la "galaxie de préfaces, d'épîtres dédicatoires et de poèmes qui créent un véritable cosmos du discours, indiquant au lecteur [...] quel rôle il doit assumer" 11 . Si nous considérons ce premier quarto, outre sept "Commendatory Verses" et dix-sept "Dedicatory Sonnets" sur lesquels s'ouvre le volume, chacun des trois livres est précédé d'un "Proem" et chaque canto d'un quatrain qui le résume. Et le volume se ferme sur "A Letter of the authors to Sir Walter Raleigh" dans laquelle Spenser décrit le plan de son poème et l'idée qui y préside ${ }^{12}$. Dans le Proem au livre 1, Spenser se situe de nouveau dans une tradition: il déclare avoir quitté ses "vêtements de berger" et avoir échangé sa "flûte de roseau" contre une "trompette héroïque". Après la pastorale, il va se tourner vers la poésie épique à l'imitation de Virgile dont il parodie l'Enéide : "Lo I the man..."13.

La qualité orale du style de Spenser a été étudiée par John Webster et Walter Ong. Tous deux soulignent que Spenser s'inscrit bien dans la tradition épique par l'abondance des "formulaic expressions", qui n'avaient pas disparu complètement avec l'apparition des "chirographic and typogaphic styles". Mais Walter Ong fait également remarquer qu'à la Renaissance, il était déjà impossible d'organiser un poème épique à la façon de jadis, ce que démontre l'écart considérable entre le but proclamé des auteurs épiques tardifs et leur réalisation, Spenser en étant le meilleur 
exemple ${ }^{14}$. En effet, Spenser doit recourir, autant par nécessité que par goût, à cette "galaxie" d'appendices pour communiquer son intention au lecteur. Dans la "Lettre à Raleigh" 15 , la première raison qu'il donne à cette nécessité d'explication est que, son poème étant ce qu'il appelle "a continued allegory or dark conceit", il veut le protéger des interprétations erronées ("misconstructions"). Et il ajoute: "The general end of all the booke is to fashion a gentleman or noble person in vertuous or gentle discipline", ce qui revient à dire que le lecteur doit pouvoir, grâce à ces explications, reconnaître des allégories morales et des allégories politiques (concernant la cour). De fait, les explications données par Spenser concernent surtout les "personae" qu'il attribue à la reine, dont la première est celle de "Reine des fées"; et les vertus que personnifient les divers chevaliers. Or ces indications sont répétées à maintes reprises dans le titre développé de chaque livre, dans le quatrain précédant chaque canto et dans le texte du poème lui-même. Le but réel de la lettre est donc ailleurs. Tout d'abord, Spenser veut se situer de nouveau dans la tradition épique, comme il l'a fait dans le Proem au livre 1, en recourant cette fois à l'énumération : il reconnaît s'être inspiré non plus seulement de Virgile mais d'Homère, des gestes médiévales (Arthur et Godfrey), de l'Arioste et du Tasse, faisant ainsi de son poème un véritable épitomé. Mais surtout il veut donner une structure à une œuvre qui n'en est qu'à son commencement et qui s'annonce immense.

Comparant l'historien au poète épique ("poet historical"), il rappelle que ce dernier commence in medias res ("thrusteth into the middest"). Le début de l'action se trouvera donc dans le dernier livre. Voici le résumé qui, selon Spenser, serait celui d'un historien ("historiographer") : la Reine des fées, célébrant sa fête annuelle de douze jours à Cleopolis, prescrit chaque jour à un chevalier une quête différente. Les aventures de ces chevaliers sont décrites en douze livres. En un autre endroit de la lettre, il déclare que ces douze chevaliers représentent les douze vertus morales selon Aristote "et les autres", et qu'il pourrait y ajouter les vertus politiques; le prince Arthur personnifie la Magnificence qui est la somme des douze autres vertus, et parcourt le monde à la recherche de la Reine des fées qu'il a vue en rêve.

Le résumé de l'action contient des contradictions. Le chiffre douze est symbolique car il ne correspond à aucune nomenclature aristotélicienne, sinon à une tradition tardive (évoquée par "et les autres"); et le nombre vingt-quatre (nombre que les critiques ont attribué aux vertus politiques, connaissant l'obsession spensérienne pour les symétries) en est encore plus éloigné ${ }^{16}$. Mais ce résumé permet de rendre le sens des péripéties directement accessible au lecteur en faisant des chevaliers (qui, apprenons-nous dès le premier livre, constituent ensemble "the Order of the Maidenhead", I.7.46) les champions de la Reine. D'autre part, Spenser exprime clairement son désir d'écrire une œuvre dont Elizabeth est le centre. Il précise que la Reine des fées représente "glory in my general intention", d'où le nom de Gloriana; mais aussi "the most excellent and glorious person of our soveraine the Queene", qu'il nomme plus loin "a most royall Queene and Empress". Il dit également vouloir représenter la reine en tant que femme sous les traits de Belphœbe, Phœbe étant, dit-il, un autre nom de Cynthia, chère à Walter Raleigh à qui la lettre est destinée. 
Cette lettre, au-delà de l'allégorie morale et politique, permet donc de comprendre le plan projeté de l'œuvre. Mais sa lecture attentive, jointe à celle des autres éléments d'accompagnement, a permis à des critiques récents de déchiffrer certains des éléments du "dark conceit". En effet, le sonnet dédicatoire à Lord Burghley, Sir William Cecil, suggère la possibilité d'une lecture plus profonde lorsque Spenser, parlant de ses "rhymes", écrit :

Yet if their deeper sence be inly wayd,

And the dim vele, with which from commune view

Their fairer parts are hid, aside be layd,

Perhaps not vain they may appeare to you .

Etant donné le destinataire du sonnet, certains critiques ont défendu l'idée d'une mystique impériale soutenue par Spenser, qui aurait voulu faire d'Elizabeth une souveraine arbitre dont le pouvoir se serait étendu sur tous les territoires où aurait régné un protestantisme tolérant ${ }^{17}$, idée déjà suggérée par Spenser dans la dédicace : "To the most High, Mightie and Magnificent Empresse". D'autres études ont porté sur un symbolisme numérologique qui, comme nous l'avons vu, est déjà apparent dans le nombre même des livres : douze ou peut-être vingt-quatre livres annoncés : six livres publiés du vivant de l'auteur et des fragments d'un septième après sa mort, chiffres sur lesquels nous reviendrons dans notre conclusion.

Mais il est possible aussi de chercher un élément de structure en dehors de la "Lettre à Raleigh" : par exemple le mode de narration symbolique et les images de production de l'œuvre. Ainsi dans le proème au livre I, Spenser, après s'être identifié à l'image d'un poète virgilien, invoque l'aide de Clio, muse de l'histoire : "Helpe then, O holy Virgin chief of nine". Le poète lui demande :

Lay forth out of thy evelasting scryne

The antique rolles, which there lye hidden still,

of Faerie Knights and fairest Tanaquil,

Whom that most noble Briton Prince so long

Sought through the world...

Et il ajoute : "O helpe thou my weake wit and sharpen my dull tongue", vers qui, dans un raccourci saisissant, télescope les images de la poésie orale et de la poésie écrite : le poète dicte ou copie le manuscrit de la muse.

Ce passage contient de nombreuses notations qui reparaîtront, contribuant à la cohérence du poème. Tout d'abord, Spenser, reprenant l'un des thèmes révélés dans la "Lettre à Raleigh", fait de la recherche de la Reine des fées par Arthur le fil conducteur majeur du poème. Tanaquil est en effet Gloriana, ainsi qu'il est révélé plus loin dans le poème (II.10.76), à l'intérieur d'un autre manuscrit sur lequel nous reviendrons. 
Le mot "scryne" qui désigne le coffret contenant ces parchemins n'apparaît que deux fois dans la Faerie Queene. Il est défini dans le dictionnaire de Cooper comme "a coffer or other like place wherein [...] secret things are kept"18. Dans une étude récente sur l'incidence qu'a eu la carrière secrétariale de Spenser sur son œuvre, R. Rambuss souligne la notion du secret. Il s'attarde sur l'expression "everlasting scryne": les révélations, toujours remises à plus tard, entre autres celles sur le personnage de Gloriana, qu'il appelle non pas le centre mais le "vanishing point" du poème, resteront à jamais non dites ${ }^{19}$. Le parchemin de Clio ne révèlera jamais tout son contenu. Il rappelle que, dès 1595 , Spenser déclarait, dans le sonnet 33 des Amoretti, qu'il n'achèverait pas la Faerie Queene :

Great wrong I do, I can it not deny,

To that most sacred Empresse my dear dred,

Not finishing her Queene of Faery,

That mote enlarge her living praises dead.

D'autre part, le parchemin contenu dans le second "scryne", le Briton Moniment (II. 9.59), intitulé aussi "A Chronicle of Briton Kings / From Brute to Uthers Rayne" (2.10. Quatrain), s'interrompt brusquement, et le prince Arthur qui le lisait ne connaîtra pas le secret de sa naissance :

After him Uther, which Pendragon hight,

Succeeding There abruptly it did end,

Without full point, or other Cesure right,

As if the rest some wicked hand did rend,

Or th'author selfe could not at least attend

To finish it....

Ce manuscrit inachevé est le reflet du manuscrit qui le contient, celui de la Faerie Queene elle-même.

L'invocation à Clio est reprise plusieurs fois au cours du récit pour rappeler cet élément de structure. Dans le troisième livre, le poète demande à la muse de l'aider à dresser l'arbre généalogique de la reine (en tant qu'héritière Tudor) depuis le mariage de Britomart et Artegall. En réponse, Spenser nous présente la chronique de Merlin, un parchemin qui s'inscrit lui aussi dans le grand manuscrit du poème :

In thy great volume of Eternity:

Begin, $O$ Clio, and recount from hence

My glorious Soveraines goodly auncestrie. (III.3.4)

Dans le quatrième livre, le poète demande à Clio son aide pour dresser le catalogue des dieux marins à l'occasion du mariage de la Tamise et de la Medway : 


\section{L'IDÉE DU LIVRE}

Helpe therefore, O thou sacred imp of Jove,

The nursling of Dame memory his deare,

To whom those rolles, layd up in heaven above,

And records of antiquity appear. (IV.11.10)

Autant que la reine, Spenser célèbre l'Angleterre en faisant la liste de ses richesses. Enfin, dans les fragments du septième livre, qui constituent un appendice où le fil du récit des six premiers livre est abandonné, Spenser demande à Clio de céder la place à Calliope, muse de la poésie épique : "O Clio, lend Calliope thy quill" (VII.6.37).

Il existe donc une cohérence dans l'introduction de manuscrits secondaires à propos desquels le poète renouvelle chaque fois son invocation, soulignant la filiation de Clio avec la mémoire. Et le goût presque obsessionnel pour les catalogues, qui dépasse la simple tradition épique, se manifeste notamment à l'occasion des deux manuscrits auxquels nous avons fait allusion à propos du second "scryne". L'épisode de leur découverte prend place à la fin de la description de la Maison de l'Âme ("the House of Alma") qui n'est autre que le corps humain. La troisième loge du cerveau est une bibliothèque gardée par le vieillard Eumnestes :

This man of infinite remembrance was, And things foregone through many ages held,

Which he recorded still, as they did pas,

Ne suffred them to perish through long eld...

But laid them up in his immortall scrine

Were they for ever incorrupted dweld ... (II.9.56)

Robert Estienne donne au mot "scrinium" le synonyme "cabinet"20. C'est le sens qu'il revêt ici :

His chamber was hangd about with rolles,

And all records from auncient time deriv'd,

Some made in books, some in long parchment scrolles,

They were all worm-eaten, and full of canker holes. (II.9.57)

C'est en ce lieu que le chevalier Guyon (la Tempérance) et le prince Arthur vont connaître chacun l'histoire de leur ascendance :

Then as they gan his librarie to view,

And antique registers for to avise,

There chaunced to the Princes hand to rize,

An auncient book, hight Briton Moniments. (II.9.59) 
Sir Guyon chaunst eke on another booke,

That hight Antiquitie of Faerie Land ... (II.9.60).

Guyon, qui appartient au Pays des fées, et le prince Arthur, qui est breton, pourront chacun lire "their countries ancestry". Le prince, avons-nous vu, sera déçu. Quant à Guyon, il prendra connaissance de l'arbre généalogique de la reine en tant que Reine des fées (Tanaquil ou Gloriana) qui fait remonter celle-ci à Prométhée et fait d'elle une candidate à l'Empire du monde.

L'une des préoccupations centrales de Spenser a été l'exploitation des possibilités du livre imprimé. Pour mieux faire comprendre l'idée de certains de ses poèmes, il a eu recours à ces "panoplies" ou "galaxies" de textes explicatifs conjoints. Nous avons vu que, dans le Proem du livre I, il avait déterminé le mode symbolique de sa narration : la Muse remettant au poète un manuscrit à l'intérieur duquel figurent d'autres manuscrits qui, souvent, établissent les justifications politiques du grand plan impérial.

Mais, à l'intérieur du récit, d'autres formes d'écrits jouent un rôle. On a pu parler du livre I de la Faerie Queene comme d'une "bataille des livres". Kenneth Gordon, dans l'article "Books in the Faerie Queene" de la Spenser Encyclopedia, en dehors de ces livres à incidence politique, distingue les livres sacrés et les livres magiques qui ont leur rôle à jouer dans l'action. Nous ne pouvons que les citer après lui : parmi les premiers, le Saveours Testament que le chevalier Redcross donne au prince Arthur (I.9.19); et le "sacred booke, with blood ywrit" de Fidelia, qu'elle seule peut lire, et qui lui permet d'entretenir Redcross "Of God, of Grace, of Justice, of Free Will" (I.10.13). Parmi les livres magiques, il y a ceux d'Archimago qui lui permettent d'évoquer des esprits "To aide his friends, or fray his enemies", et de faire croire à Redcross qu'Una (personnification, entre autres, de l'Eglise protestante) est impure (I.1.36-8); et ceux de l'enchanteur Busirane, qualifiés de "wicked" (III.12.31-2), que ce dernier emploie pour séduire une héroïne, Amoret, "with the literalized conceits of courtly love".

Ces livres magiques évoquent peut-être certaines croyances ou rituels catholiques, rappelant ces "bookes and papers" que vomit le dragon "Error" frappé à mort par Redcross (I.1.20). Comme le fait remarquer Kenneth Gross, ces livres, religieux ou magiques, "are codices rather than scrolls, whether they are manuscripts or printed copies is not clear"21. Mais ce problème rejoint sans doute celui de l'intemporalité du Pays des fées, où coexistent Gloriana et le prince Arthur $^{22}$. Le même auteur fait également remarquer que les images du livre disparaissent pratiquement vers la fin du poème. Mais est-ce l'amour frustré de Spenser pour les livres qu'il a cessé d'évoquer ouvertement qui, dans le livre VI, lui fait choisir des héros portant le nom de Aldus, Aldine et Calepine, noms évoquant un éditeur vénitien et l'auteur d'un célèbre dictionnaire. Plusieurs critiques se sont posé la question ${ }^{23}$.

Autre manifestation intéressante de l'idée qui a présidé à l'élaboration de la Faerie Queene, la structure fondée sur la symbolique des nombres. Habilement, dans la lettre à Raleigh, Spenser commence par suggérer des nombres pouvant 


\section{L'IDÉE DU LIVRE}

évoquer des concepts qu'il désire voir associés à son poème. Il suggère d'abord le nombre douze (douze livres divisés en douze cantos) qui évoque le cycle fermé du zodiaque, comme c'était déjà le cas pour le Shepeardes Calender Conteyning Twelve Aeglogues proportionable to the twelve monethes, mais aussi dans le cas de modèles tels que Virgile et Stace. D'autre part, douze chevaliers autour de la personne de Gloriana font d'elle le "jour spirituel", au même titre que le Christ entouré des douze apôtres ${ }^{24}$. Enfin, douze est également un nombre lié au nom $\mathrm{d}^{\prime} \mathrm{Arthur}^{25}$. Spenser laisse entendre ensuite qu'il écrira vingt-quatre livres si les douze premiers sont appréciés, nouveau nombre qui évoque le cycle du jour et de la nuit, sur lequel se fondent aussi l'Iliade et l'Odyssée qui comportent ce nombre de "chants"; nombre qui évoque aussi d'autres éléments très proches de la pensée de Spenser : par exemple les vingt-quatre théorèmes de la Monas Hieroglyphica de John Dee qui, comme Spenser, soutenait l'idéal impérial ${ }^{26}$; ou les vingt-quatre places autour de la Table Ronde, conservée à Winchester, dont les divisions avaient été peintes sous les Tudors pour représenter les places de vingt-quatre chevaliers, c'est-à-dire autant qu'il y avait de chevaliers de la Jarretière, et souligner l'origine commune des deux mythes dont ils se réclamaient ${ }^{27}$. Remarquons enfin que le cinéaste Peter Greenaway attribue vingt-quatre livres à Prospero dans son film Prospero's Books, peut-être à partir de ses souvenirs d'université 28 .

Mais Spenser n'écrivit que six livres et des fragments d'un septième. Le lecteur avait lu les six premiers livres en ayant cette richesse symbolique des nombres présente à l'esprit. L'abandon du poème, annoncé dans le sonnet 33 des Amoretti, imposait une nouvelle structure. Le symbolisme le plus évident du nombre six est celui de la création du monde. Or l'équation entre le poète et le Créateur, reposant sur l'étymologie du verbe grec "poein", "faire", était familière à Spenser qui emploie "make" dans le sens de "versify"29. Il avait été de plus l'ami de Philip Sidney qui développe cette image dans sa Defence of Poesie $e^{30}$. Michael Murrin écrit: "The men of the Renaissance believed that the cosmos itself came into being by the spoken word of the Lord, just as the poet creates fictional worlds through speech"31.

Que Spenser n'ait écrit que six livres pourrait être considéré comme une coïncidence signifiante si Spenser, ou le nouvel éditeur du folio de $1609^{32}$, n'avait fait en sorte que les fragments du septième livre s'achèvent sur l'expression d'un désir de repos du poète, et ne se closent sur le mot hébreu "Sabaoth" : "O that great Sabbaoth God, grant me that Sabaoths sight" (VII.8.2). Comme le Créateur, Spenser aspire au repos du septième jour après la création de son propre cosmos. Mais une autre intention ou une autre coïncidence symbolique a pu présidé au choix du mot "Sabaoth" car le nom d'Elizabeth, point central ou "point de fuite" du poème, signifie étymologiquement "repos du Seigneur" 33 .

Bernard TANNIER Université de Villetaneuse 
Philip Sidney, A Defence of Poetry, Oxford U. P., 1975, p.24.

2 Michael McCanles, "The Shepheardes Calender as document and monument", Studies in English Literature 1500-1900, 22, 1982. A. Hadfield, Literature, Politics and National Identity, Cambridge U.P., 1994, p.174 : "In its physical form, the poem accompanied by a whole panoply of explanations, glosses and commentary so that it appears as a 'fictional imitation of a humanist edition of classical texts', suggested it was a deliberate ploy".

3 McCanles, op.cit, p. 14. Walter Ong, Ramus, Method and the decay of Dialogue, Harvard, 1974, (Reprint : Octagon Books) pp. 313-14.

4

McCanles, op. cit., p. 8.

5 Ruth S. Luborsky, "The Allusive Presentation of the Shepheardes Calender" in Spenser Studies I, ed. P. Cullen and T.P. Roche, U. of Pittsburgh Press, 1980, pp. 7-8 et 26-7.

6 The Spenser Encyclopedia, ed. A. C. Hamilton, U. of Toronto Press, 1990, pp. 737-8.

$7 \quad$ Three Proper, and Witty Familar Letters : lately passed between Two University Men. Two Other, very Commendable Letters, of the same Mens Writing, London, 1580.

8 "Nine Comoedies named for the nine Muses in imitation of Herodotus" : voir The Spenser Encyclopedia, p. 739.

9 W. Wilson, The Poetry of Edmund Spenser : A Study, Columbia U. P., 1965, p. 33 : "No English poet had ever been announced so pretentiously".

10 Spenser semble avoir encouru le déplaisir de Sir William Cecil pour avoir pris le parti de Leicester qui était opposé au mariage entre la reine et le duc d'Alençon; et pour avoir caricaturé ce dernier dans Mother Hubberds Tale (publié plus tard dans les Complaints).

11 Walter Ong, Interfaces of the Word, Cornell U. P., 1977, p. 76.

12 Maureen Quilligan, The Language of Allegory, Cornell U. P. 1979, p. 232 : "I would guess that Spenser appended the quatrains to satisfy editorial as opposed to poetic demands, just as he wrote the open 'Letter to Raleigh' to answer the 


\section{L'IDÉE DU LIVRE}

printer's question about 'how doubtfully all allegories mays be construed'... It is as if Spenser, asked to function as an allegorical critic of his own poem, neglects the allegory for the allegoresis".

13 "Ille ego... ", vers ajoutés, à la Renaissance, au début de l'Enéide et que l'on croyait être de Virgile. Cf. Edmund Spenser, The Faerie Queene, ed. A.C. Hamilton, Longman Annotated Poets, London, 1977, p. 27.

14 Walter Ong, Interfaces of the Word, pp.196-99. John Webster, "Oral form and written craft in Spenser's Faerie Queen", Studies in English Literature 15001900, 16, 1976.

15

The Faerie Queene, pp. 737-8.

16 A. Fowler, Spenser and the Numbers of Time, London, 1964. Sur la tradition de douze vertus, Fowler renvoie à un article de V.B. Hulbert qui la fait remonter à Thomas d'Aquin (in The Works of Edmund Spenser, a Variorum Edition, ed. Osgood and Lotspeich, Baltimore, Vol.1, 1932, pp. 353-56). Hulbert écrit pourtant : "There is, of course, the alternate explanation that Spenser's decision to have twelve books (the standard number for an epic) may have had nothing to do with the twelve Aristotelian virtues; i.e. the fact that there are twelve virtues in the Faerie Queene would naturally be the result of an arrangement in twelve books, each of which deals with a virtue" (p. 357). Il cite également R. Ellrodt, Neoplatonism in the Poetry of Spenser, Genève, E.Droz, 1960, p. 110. Sur le nombre vingt-quatre, voir Michel Leslie, Spenser's Fierce Warres and Faitfhull Loves, Martial and Chivalric Symbolism in the 'Faerie Queene', Cambridge, 1983, pp. 188-9.

17 Frances Yates, Astrcea, the Imperial Theme in the Sixteenth Century, London, 1975. Douglas Brookes-Davies, The Mercurian Monarch, Magical politics from Spenser to Pope, Manchester, 1983.

The Faerie Queene, p. 28.

19 Richard Rambuss, Spenser's Secret Career, Cambridge U. P., 1993, pp. 667. Rambuss cite D.L. Miller, The Poem's Two Bodies : the Poetics of the 1590 'Faerie Queene', Princeton, 1988, p.138.

Ibid., p. 66.

Spenser Encyclopedia, pp. 103-4. 
22 Sur la distinction entre Britons and Elves, voir Isabel Rathbone, The Meaning of Spenser's Faeryland, New York, 1965, (first edition, 1937).

23 James Nohrnberg, The Analogy of the 'Faerie Queene', Princeton, 1976, p. 683. Harry Berger, "A Secret Discipline", in William Nelson, Form and Convention in the Poetry of Edmund Spenser, Columbia U. P., 1961, p. 171, note 6.

24 V.H. Hopper, Medieval Number Symbolism, Columbia, 1938, p. 86, qui cite saint Augustin, Commentaires sur saint Jean, 49.8.

25

Michael Leslie, op.cit., pp. 187-88.

26 M.T. Walton, "Johne Dee's Monas Hieroglyphica: Geometrical Cabala", Ambix, 23, 1976. F. Yates, The Occult Philosophy in the Elizabethan Age, Londres, 1979, pp .79-108.

27 Michael Leslie, op. cit., p. 188.

28 Peter Greenaway, Prospero's Books, a Film of Shakespeare's 'The Tempest', London, 1991. "All of the twenty-four volumes [...] made Prospero so powerful he could command the dead and make Neptune his servant", p. 12.

29 The Works of Edmund Spenser, a Variorum Edition, Vol. VII, pp. 62 et 32021. Cf. Shepheardes Calender, "June", vers 82.

30 Philip Sidney, op. cit, .p. 22.

31 Michael Murrin, The Veil of Allegory, U.of Chicago Press, 1969, p. 66.

32 Les deux quartos, de 1590 et 1596, avaient été publiés par Ponsonby. Le folio de 1609 indique : "Printed by H. L. for Mathew Lownes".

33 A.C. Hamilton, "Our new poet: Spenser, "welle of English undefiled' ", in $A$ Theatre for Spenserians, ed. M.J. Kennedy and J.A. Reither, Manchester U. P., 1969, p. 110. Hamilton a trouvé cette étymologie dans Remains Concerning Britain de William Camden, 1605. 